\title{
ARTí́CUlO
}

\section{Situación actual del recurso pepino de mar en el Sistema Arrecifal Veracruzano, México}

Current status of the sea cucumber resource in the Sistema Arrecifal Veracruzano, Mexico

\author{
Elizabeth Romero-Hernández ${ }^{1}$, Pedro C. Reyna-González ${ }^{*}$ \\ y Guadalupe Pantoja-Yepez ${ }^{1}$
}

'Instituto Nacional de Pesca y Acuacultura-Veracruz, Av. Ejército mexicano 106, Col. Ex Hacienda Ylang Ylang, Boca del Río, Veracruz, C.P. 94298, México. *Autor corresponsal: pedroreynaglez@gmail.com

\begin{abstract}
Throughout snorkeling and scuba diving ten study zones (sampling banks established in previous research by Instituto Nacional de Pesca - INAPESCA) were evaluated where the presence of the sea cucumber was reported. By line transect sea cucumbers were censed and collected to be measured and weighed, as well as their habitat characteristics were recorded. A total of 138 stations in the Sistema Arrecifal Veracruzano (SAV), equivalent to $37.794 \mathrm{~m}^{2}$ of reef area sampled in the north and south were analyzed. In just 25 sampling points there were found Holothuria floridana (associated to seagrass and sand habitat, 0-1.5 $\mathrm{m}$ deep, with total length average of $10.88 \mathrm{~cm}$ and fresh weight average of $64.09 \mathrm{~g}$ ) and Isostichopus badionotus (associated to sand and live coral habitat, $1.5-15 \mathrm{~m}$ deep, total length average of $27.2 \mathrm{~cm}$ and fresh weight of $769.19 \mathrm{~g}$ ). The average density of the total banks evaluated was 0.008 ind $\mathrm{m}^{-2}$. Results indicate that sea cucumber population in SAV is fragmented (patchiness or aggregation sites) and is not susceptible of exploitation (low density of individuals per unit area). As a management measure, some banks were suggested as potential sites in which there was a major presence of sea cucumber, with the purpose of monitoring these areas and evaluate the density of the population. This research and its results offer a current status about the fishing potential of the sea cucumber so that in the future, it will allow the suggestion to investigate their culture for the natural repopulation for a sustainable use of this marine resource in the region.
\end{abstract}

Key words: Coral reefs, density, population, sea cucumber, SAV

Resumen.- Se evaluaron 10 zonas de estudio (bancos de muestreo establecidos en investigaciones previas por el Instituto Nacional de Pesca - INAPESCA) mediante buceo libre y autónomo, donde se registró la presencia de pepino de mar. Usando el método de transecto en banda, se censaron y recolectaron los pepinos para luego medirlos y pesarlos. Se registraron además las características del hábitat. Se analizaron un total de 138 estaciones equivalentes a $37.794 \mathrm{~m}^{2}$ de área muestreada en arrecifes de la zona norte y sur del Sistema Arrecifal Veracruzano (SAV). En solo 25 estaciones se encontró pepino de mar de las especies Holothuria floridana (asociada al hábitat de pastos marinos y arena, a profundidades de 0-1,5 m, longitud total promedio de $10,88 \mathrm{~cm}$ y peso fresco promedio de $64,09 \mathrm{~g}$ ) e Isostichopus badionotus (asociada al hábitat de arena y corales vivos, profundidades de 1,5 a $15 \mathrm{~m}$, longitud total promedio de $27,2 \mathrm{~cm}$ y peso fresco promedio de $769,19 \mathrm{~g}$ ). La densidad promedio en el total de los bancos fue de 0,008 ind. $\mathrm{m}^{-2}$. Los resultados indican que la población de pepino de mar en el SAV se encuentra fragmentada (distribución en parches o sitios de agregación) y no es susceptible a explotación (baja densidad por unidad de área). Como una medida de manejo, se proponen algunos bancos como sitios potenciales en los cuales hubo mayor presencia de pepino de mar, con la finalidad de monitorear estas áreas y evaluar la densidad de la población. Esta investigación muestra el panorama actual acerca del potencial pesquero del pepino de mar para que en el futuro, se investigue su cultivo para repoblamiento natural, permitiendo un aprovechamiento sustentable de este recurso en la región.

Palabras clave: Arrecifes coralinos, densidad, población, pepino de mar, SAV

\section{INTRODUCCIÓN}

Los pepinos de mar son equinodermos de la clase Holothuroidea (Herrero-Pérezrul \& Reyes-Bonilla 2008, Tuz-Sulub \& Aguilar Perera 2011) que desde el punto de vista ecológico, desempeñan un papel fundamental en las comunidades bentónicas, al actuar como recicladores de nutrientes y agentes de bioturbación (Uthicke 2001), permitiendo el movimiento y oxigenación de los suelos y evitando la estratificación de los fondos (Navarro et al. 2013), convirtiéndolos en organismos indispensables para el funcionamiento de los ecosistemas marinos (Herrero-Pérezrul et al. 1999, Navarro et al. 2013).

A nivel mundial, desde el punto de vista comercial, este recurso pesquero (trepang, beché de mer), ha adquirido un 
importante interés por el alto valor económico que representa su venta (Purcell 2010), ya que el precio en el mercado se encuentra entre los 300 a 500 dólares por $\mathrm{kg}$ en presentación seco (Purcell 2010, Purcell et al. 2012). Además, existe una gran demanda por parte del mercado asiático, debido a que las poblaciones naturales de pepino de mar se encuentran sobreexplotadas, ya que este ha sido utilizado como alimento, para producción de fármacos, complejos vitamínicos y suplementos de minerales (Conand 2006, Purcell et al. 2012).

La sustentabilidad en la pesca de este recurso es de gran relevancia, ya que este organismo es vulnerable a la sobrexplotación debido a que sus poblaciones forman densas agregaciones, tienen escasa movilidad, lento crecimiento, gran longevidad y baja mortalidad natural (Purcell 2010, Anderson et al. 2011). A principios de 1990, en la Península de Baja California, se capturaban dos especies de pepino de mar (Parastichopus parvimensis e Isostichopus fuscus) con permisos de pesca de fomento (Singh-Cabanillas \& VélezBarajas 1996); sin embargo, a partir de 2003 se inició la explotación comercial en el Pacífico mexicano de la especie $I$. fuscus con esquemas de aprovechamiento para mantener la población (Reyes-Bonilla \& Herrero-Perezrul 2003, SolísMarín et al. 2013).

En 2005 se solicitan los primeros permisos en el Golfo de México, para el aprovechamiento de las especies Isostichopus badionotus, Astichopus multifidus y Holothuria floridana (Rodríguez-Gil 2007, Cervera-Cervera et al. 2008, INAPESCA 2012 1 ), no siendo hasta el 2015, cuando se publica el plan de manejo oficial de pepino de mar en la península de Yucatán (DOF 2015) ${ }^{2}$, que muestra los esquemas de manejo para la pesquería del este recurso (cuota de captura, talla mínima, periodo de veda y sitios de pesca), los cuales se basan en los informes anuales entregados por los permisionarios, que incluyen entre otros: bitácoras de pesca para evaluar la captura, estructura de tallas, pesos y estimaciones de biomasa.

En la zona litoral marina de Veracruz, se han descrito 116 especies de equinodermos que incluyen estrellas de mar, crinoideos, erizos, ofiuros y holoturoideos (Durán-González et al. 2005, Solís-Marín et al. 2014). En el caso particular del Sistema Arrecifal Veracruzano (SAV), se han reportado las especies de pepino de mar Isostichopus badionotus, Holothuria floridana, Actinopyga agassizii, Holothuria arenícola, Holothuria mexicana, Holothuria grisea, Holothuria surinamensis y Eostichopus arnesonii (DuránGonzález et al. 2005, Solís-Marín et al. 2007), las cuales son de interés comercial a nivel mundial (Purcell et al. 2012).

A pesar de que existen investigaciones sobre la biología y taxonomía de estos organismos en el SAV (Solís-Marín et al. 2007), aún no existen estudios dirigidos al aprovechamiento y conocimiento del estado actual de la pesquería de pepino de mar en este sistema arrecifal. En el 2006, ante la Comisión Nacional de Acuacultura y Pesca (CONAPESCA), se solicitó el primer permiso de pesca de fomento para la extracción de $I$. badionotus y $H$. floridana frente a las costas de Veracruz y Alvarado, incidiendo directamente en el SAV.

Por lo tanto, el objetivo de este trabajo fue evaluar, en una primera aproximación el potencial pesquero del pepino de mar con la finalidad de proveer información sobre su distribución y densidad dentro del SAV. Debido a la importancia que posee este recurso a nivel local y regional, los resultados de esta investigación muestran el panorama actual para establecer medidas de manejo para una explotación sustentable de esta pesquería y/o proponer alternativas para su conservación y repoblamiento.

\section{MATERIALES Y MÉTODOS}

El Sistema Arrecifal Veracruzano(SAV), se localiza en el estado de Veracruz, México, frente a los municipios de Veracruz, Boca del Río y Alvarado (Fig. 1). En 1992, el SAV fue declarado como Área Natural Protegida con carácter de Parque Nacional, con una extensión de 52,23 x $10^{3}$ ha (DOF 1992) ${ }^{3}$. En decreto actual, está conformado por una poligonal con una superficie total de $65,51 \times 10^{3}$ ha constituido por 28 formaciones arrecifales, incluyendo 6 islas y 2 bajos arenosos (DOF 2012, CONANP 20145).

\footnotetext{
${ }^{1}$ INAPESCA. 2012. Propuesta del plan de manejo de la pesquería de pepino de mar en la Península de Yucatán (Documento interno), 65 pp. Instituto Nacional de Pesca, Centro Regional de Investigación Pesquera, Yucalpetén, Yucatán.

${ }^{2}$ DOF. 2015. Acuerdo por el que se da a conocer el plan de manejo pesquero de pepino de mar café (Isostichopus badionotus) y lápiz (Holothuria floridana) en la península de Yucatán. Diario Oficial de la Federación. México, D.F. 12 de Mayo de 2015.

${ }^{3}$ DOF. 1992. Decreto por el que se declara área natural protegida con el carácter de Parque Marino Nacional, la zona conocida como Sistema Arrecifal Veracruzano, ubicada frente a las costas de los municipios de Veracruz, Boca del Río y Alvarado en el estado de Veracruz Llave. Diario Oficial de la Federación. México, D.F. 24 de Agosto de 1992.

${ }^{4}$ DOF. 2012. Decreto que modifica el diverso por el que se declara Área Natural Protegida, con el carácter de Parque Marino Nacional, la zona conocida como Sistema Arrecifal Veracruzano, ubicada frente a las costas de los municipios de Veracruz, Boca del Río y Alvarado del Estado de Veracruz, llave. Diario Oficial de la Federación. México, D.F. 29 de Noviembre de 2012.

${ }^{5}$ CONANP 2014. Anteproyecto Programa de Conservación y Manejo Parque Nacional Sistema Arrecifal Veracruzano, Documento de Consulta Pública, 213 pp. Comisión de Áreas Naturales Protegidas, Veracruz.
} 


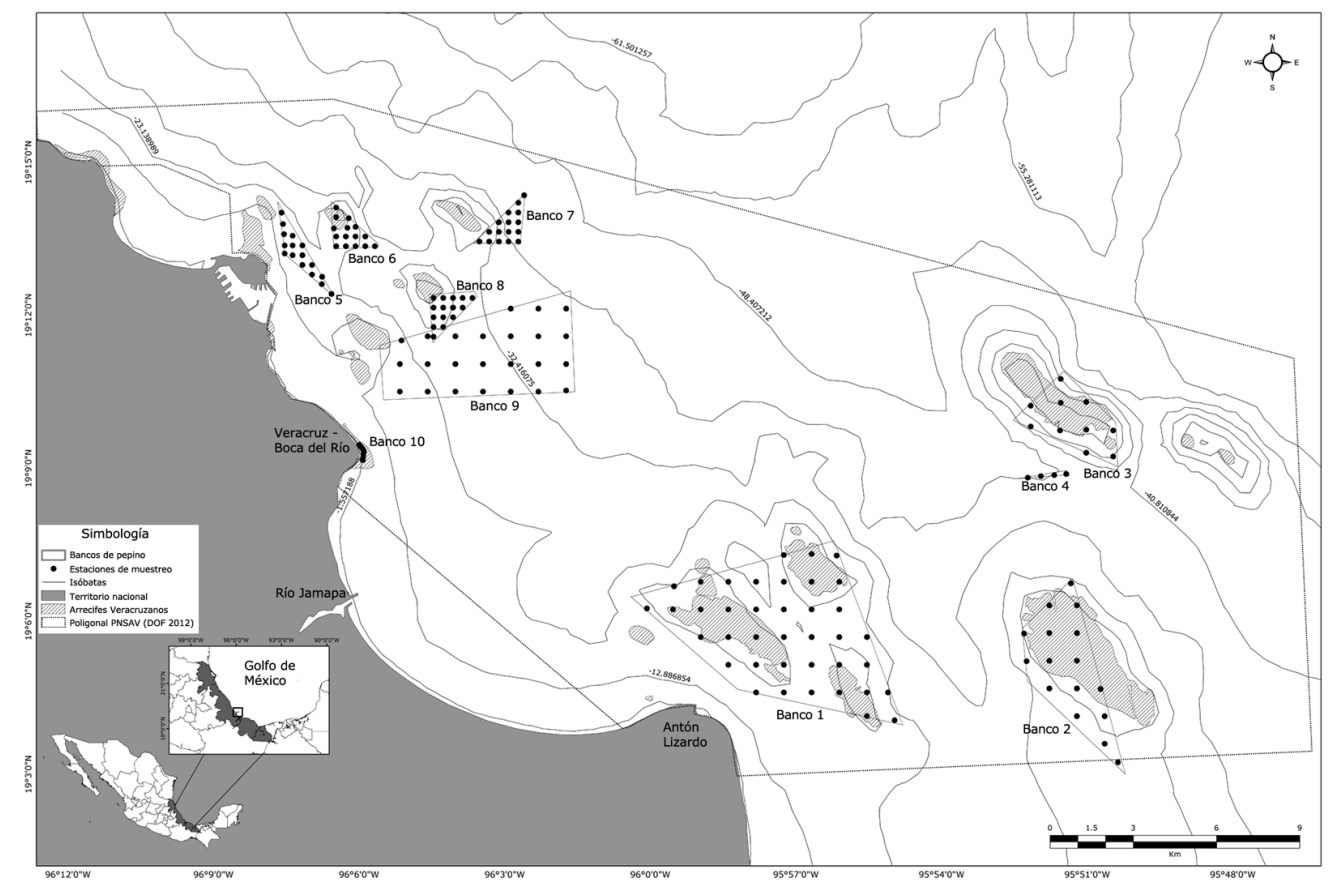

Figura 1. Sistema Arrecifal Veracruzano (SAV): en líneas punteadas se observan los bancos establecidos con presencia de pepino de mar y los puntos representan las estaciones de muestreo / Sistema Arrecifal Veracruzano (SAV): Dotted lines shows the banks established with presence of sea cucumber and dots represented the sampling stations

Este sistema se encuentra separado geográficamente en 2 complejos arrecifales: zona norte y zona sur (Lara et al. 1992, Tunnell Jr et al. 2007). La zona norte la constituyen 13 arrecifes, localizados frente a las ciudades de Veracruz y Boca del Río. En la zona sur, se encuentran 15 arrecifes ubicados frente a Antón Lizardo, a $20 \mathrm{~km}$ al suroeste del Puerto de Veracruz (DOF 20124). Ambos complejos están divididos naturalmente por la descarga del río Jamapa en la porción central del sistema e influenciado por los ríos La Antigua al norte y Papaloapan al sur (Krutak 1997, Salas-Pérez \& Granados-Barba 2008). El clima en la región es de tipo AW' $\mathrm{Z}$ (w)(i) caliente sub-húmedo con lluvias en verano, con 3 temporadas principales del año: La temporada de 'nortes' que abarca desde octubre a abril y se caracteriza por una escasa precipitación, baja temperatura ambiental e incremento de masas de aire frío proveniente del Norte, cuya fuerza puede provocar desde vientos frescos, violentos y huracanados (Zavala-Hidalgo et al. 2003, SalasPérez \& Arenas-Fuentes 2011). Una corta temporada de secas (de abril a mayo) se caracteriza por presentar elevadas temperaturas y baja precipitación (Salas-Pérez \& GranadosBarba 2008), y la temporada de lluvias, la cual se extiende de junio a septiembre y se caracteriza por temperaturas ambientales elevadas, vientos débiles que predominian del este y una alta precipitación pluvial, que alcanza sus valores máximos desde junio a agosto (Gutiérrez de Velasco \& Winant 1996, SalasPérez \& Granados-Barba 2008).

Para esta investigación, se establecieron 162 estaciones de muestreo distribuidas en 10 diferentes bancos, localizados en los arrecifes de las zonas norte y sur del SAV (Fig. 1). Se determinó un diseño de muestreo aleatorio simple (Cochran 1977, Cadima et al. 2005) durante mayo a noviembre de 2013, abarcando las 3 temporadas climáticas que inciden en el SAV (secas, lluvias y nortes) (Zavala-Hidalgo et al. 2003, SalasPérez \& Granados-Barba 2008, Salas-Pérez \& ArenasFuentes 2011). De manera sistemática, se asignó un determinado número de estaciones con base en el tamaño de cada banco y la superficie arrecifal, descartando 3 bancos (número 04, 07 y 09) debido a que en los 2 primeros, la profundidad es mayor a $30 \mathrm{~m}$ y representaba un riesgo para los buzos por el síndrome de descompresión (Desola-Alà 1990) y en el tercer banco (no. 9), se encuentra el canal de navegación (SCT 2012) ${ }^{6}$, que es la ruta de entrada y salida de embarcaciones de gran calado al puerto de Veracruz, lo cual es peligroso para la integridad de los buzos durante la investigación.

${ }^{6}$ SCT. 2012. Programa maestro del desarrollo portuario de Veracruz 2011-2016 <http://www.puertodeveracruz.com.mx/quienes-somos/ programa-maestro-de-desarrollo-portuario-2011-2016/> 
Cada unidad de muestra consistió en un transecto lineal (Skewes et al. 2002, Ramírez-González 2006) de 100 m de largo por $2 \mathrm{~m}$ de ancho para tener una superficie analizada de $200 \mathrm{~m}^{2}$ (INAPESCA 2012) ${ }^{2}$, registrando el punto inicial y final de cada transecto con ayuda de un GPS Garmin modelo 76Cx. Para los sitios de difícil acceso en embarcación (lagunas arrecifales con una profundidad menor a $1 \mathrm{~m}$ ), el muestreo se modificó realizando recorridos de distancia variable (Purcell et al.2009) de $20 \mathrm{~min}$ de nado aproximadamente a lo largo de la laguna. Se evaluó de manera relativa, el porcentaje de la cobertura de componentes abióticos (arena, coral muerto, pedacería) y bióticos (algas, corales, esponjas, pastos) de cada transecto, utilizando la escala estandarizada para la evaluación visual de la cobertura bentónica en arrecifes coralinos establecida por Hill \& Wilkinson (2004). Con ayuda del software STATISTICA Version 7.0 (Statsoft 2007) ${ }^{7}$, se realizó la prueba de Anderson-Darling para corroborar la normalidad del conjunto de la datos de la muestra (Stephens 1974, FlowersCano et al. 2014) y posteriormente, el coeficiente de correlación de Spearman (Martínez-Ortega et al. 2009) entre los organismos censados y los elementos bióticos y abióticos presentes en el muestreo.
Se recolectaron muestras biológicas e identificaron las especies de pepino de mar (Purcell et al. 2012), los cuales se mantuvieron en un contenedor con agua salada y hielo para su conservación. Los organismos capturados fueron medidos y pesados (longitud dorsal, ancho total, peso fresco y peso eviscerado), con ayuda de una cinta métrica y una balanza digital con una precisión de $0,1 \mathrm{~g}$. Posteriormente, se determinó el sexo con base en la morfología de los túbulos de la gónada de acuerdo a Ramofafia et al. (2000) y Fajardo-León et al. (2008).

Para obtener la distribución espacial de los organismos en los bancos evaluados se utilizó el método geoestadístico de interpolación espacial (estimación de puntos de referencias) o kriging ordinario (Moral-García 2004) a través del software ArcMap $^{\text {TM }}$ Versión 9.3 (ESRI 2009). Se contabilizó el número de ejemplares totales de cada banco para calcular la abundancia en términos de densidad (Martella et al. 2012) y estimar la abundancia promedio (número de organismos encontrados por unidad de área muestreal) y la abundancia poblacional (número de organismos estimados por unidad de área de cada banco). Se obtuvo la estimación de los parámetros por intervalos de acuerdo con Anderson et al. (2008) para comparar las densidades entre los diferentes bancos del recurso pepino de mar dentro del SAV.

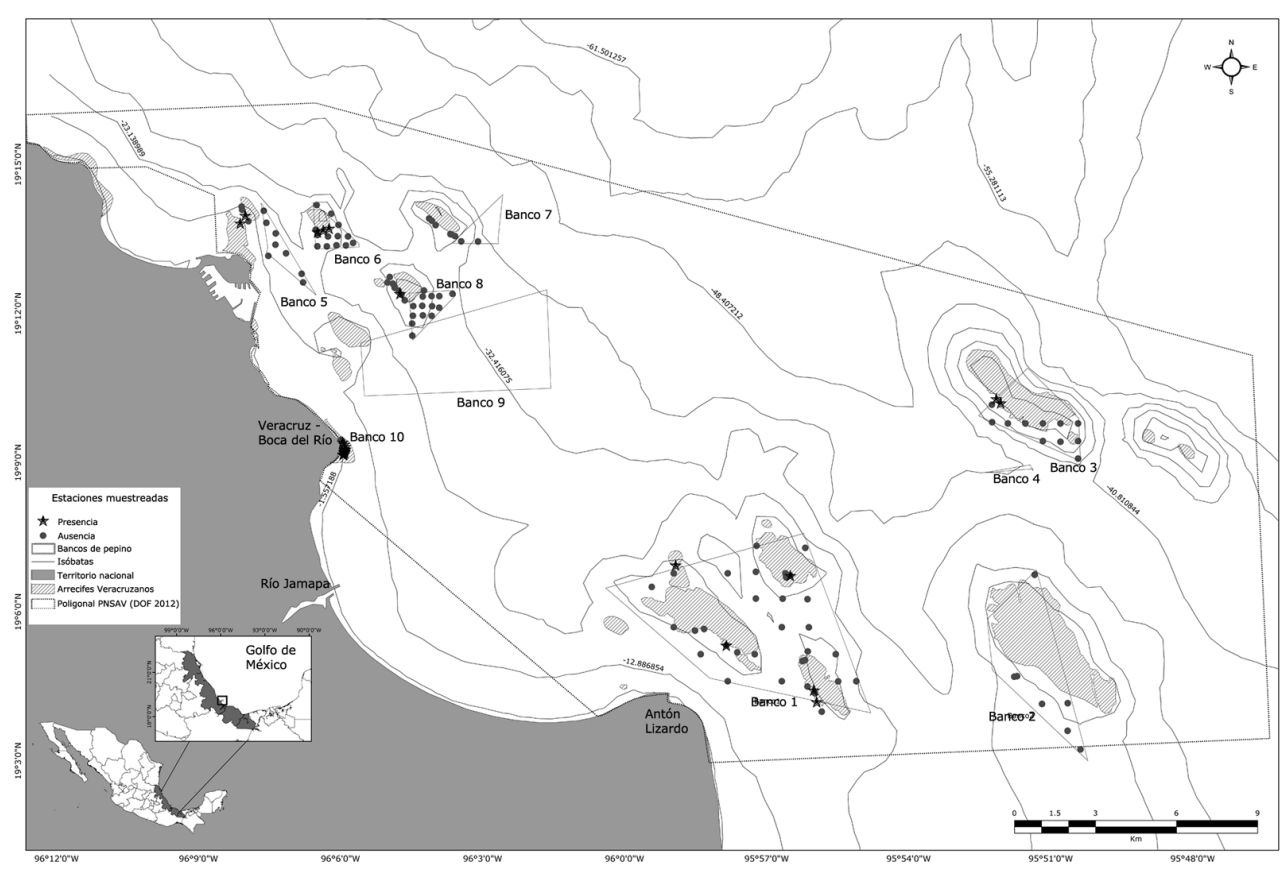

Figura 2. Estaciones visitadas durante los muestreos en las cuales se encontró pepino de mar / Stations visited during the sampling, where sea cucumber was found

${ }^{7}$ Statsoft 2007. Statistica software. CD Version 7 para Windows. 


\section{Resultados}

Se muestrearon un total de 138 estaciones, equivalente a 37.794 $\mathrm{m}^{2}(85,18 \%)$ del número total de sitios establecidos. En solo 25 de estas estaciones (18\%), distribuidas en 7 diferentes bancos, se encontraron pepinos de mar (Fig. 2). La profundidad (> $30 \mathrm{~m}$ ) y el acceso al arrecife en zonas muy someras y de alto oleaje, limitaron el número de las estaciones muestreadas.

Se obtuvo una muestra total de 45 ejemplares de pepino de mar durante los muestreos, 35 correspondieron a Holothuria floridana (encontrado en sitios con arena y camas de pastos marinos, en profundidades entre 0,6 y 1,5 m) y 10 a Isostichopus badionotus (en zonas de arena con coral vivo que utilizan como refugio, en profundidades entre 1,5 y $15 \mathrm{~m}$ ). También se observó Pseudothyone belli; sin embargo, esta especie críptica no es de interés comercial por lo que no fue recolectada.

Tabla 1. Estadísticas descriptivas de las biometrías tomadas al pepino de mar recolectados en los bancos del Sistema Arrecifal Veracruzano / Descriptive statistics of biometrics of sea cucumbers collected in Sistema Arrecifal Veracruzano's banks

\begin{tabular}{|c|c|c|c|c|}
\hline & $\begin{array}{l}\text { Longitud } \\
\text { total }(\mathrm{cm})\end{array}$ & $\begin{array}{l}\text { Ancho } \\
(\mathrm{cm})\end{array}$ & $\begin{array}{c}\text { Peso fresco } \\
\text { (g) }\end{array}$ & $\begin{array}{c}\text { Peso } \\
\text { eviscerado }(\mathrm{g})\end{array}$ \\
\hline \multicolumn{5}{|c|}{ Holothuria floridana (Pourtalès, 1851)* } \\
\hline Mínimo & 6,00 & 1,00 & 15,56 & 4,74 \\
\hline Máximo & 17,00 & 5,00 & 159,84 & 44,19 \\
\hline Media & 10,88 & 2,57 & 64,09 & 24,65 \\
\hline Varianza (n-1) & 6,04 & 0,66 & 782,59 & 85,32 \\
\hline Desviación típica (n-1) & 2,46 & 0,81 & 27,97 & 9,24 \\
\hline \multicolumn{5}{|c|}{ Isostichopus badionotus (Selenka, 1857)** } \\
\hline Mínimo & 18,00 & 6,00 & 301,09 & 222,24 \\
\hline Máximo & 37,00 & 12,00 & $1.127,35$ & 648,56 \\
\hline Media & 27,20 & 8,43 & 769,19 & 457,02 \\
\hline Varianza (n-1) & 27,68 & 2,08 & $52.439,78$ & $15.931,86$ \\
\hline Desviación típica (n-1) & 5,261 & 1,44 & 229,00 & 126,22 \\
\hline
\end{tabular}

*35 número de observaciones

**10 número de observaciones
La talla promedio de $H$. floridana fue de $10,88 \pm 2,46 \mathrm{~cm}$ y el peso fresco promedio de $64,09 \pm 27,17 \mathrm{~g}$; por sus características morfológicas (línea lateral de tetinas) se determinó que son organismos adultos. I. badionotus presentó talla promedio de $27,2 \pm 5,26 \mathrm{~cm}$ y peso fresco promedio de 769,19 $\pm 229,00 \mathrm{~g}$, siendo también organismos adultos. Debido a que los organismos encontrados fueron pocos, no se considera una muestra representativa, por lo que no es posible obtener una estimación real de la estructura de tallas, pesos y biomasa (Tabla $1)$.

En las estaciones de muestreo se presentó una gran variabilidad de los datos registrados (temperatura, visibilidad, profundidad, número de organismos y la cobertura de cada estación). En la Tabla 2 se muestran los estadísticos descriptivos de cada una de las variables registradas. La prueba de Anderson-Darling mostró que los datos no se distribuyen de manera normal (Tabla 3). Se detectó una correlación moderada entre el entre el número de organismos, principalmente de la especie $H$. floridana y los pastos marinos presentes $(\mathrm{r}=0,6, P$ $=0,001)$; no se detectó una relación significativa $(\mathrm{r}=-0,5, P=$ 0,26 ) entre la profundidad y el número de organismos (Tabla 4); bajo los $20 \mathrm{~m}$ de profundidad no se encontró ningún espécimen.

Los resultados obtenidos muestran que no existe una distribución homogénea del recurso pepino de mar en los bancos muestreados en el SAV. En algunos bancos, fueron encontrados de 1 a 6 individuos, mientras que en algunas otras estaciones del mismo banco, no se encontró ningún organismo. En el banco 10 (Fig. 2), se observó una mayor cantidad del recurso pepino de mar (principalmente de la especie $H$. floridana), con un intervalo para la media de $0,071 \pm 0,039$ ind. $\mathrm{m}^{-2}(\operatorname{Linf}=0,031 \mathrm{Lsup}=0,110)$ con un nivel de confianza de 95\%; no obstante, la densidad promedio en los bancos muestreado del SAV es muy baja, estimándose una densidad total de 0,008 ind. $\mathrm{m}^{-2}$ (Tabla 5).

Tabla 2. Estadística descriptiva de las variables registradas durante la evaluación en los bancos de pepino de mar muestreados / Descriptive statistics between the variables recorded during the evaluation in the sampled sea cucumber Banks

\begin{tabular}{lcccccccccccc}
\hline & $\begin{array}{c}\text { Prof } \\
(\mathrm{m})\end{array}$ & $\begin{array}{c}\mathrm{V} \text { is } \\
(\mathrm{m})\end{array}$ & $\begin{array}{c}\text { Tem } \\
\left({ }^{\circ} \mathrm{C}\right)\end{array}$ & $\begin{array}{c}\text { Num } \\
\text { org. }\end{array}$ & Pas & Alg & Coral & Esp & Are & Limo & Ped & CM \\
\hline No. de observaciones & 138 & 138 & 138 & 138 & 138 & 138 & 138 & 138 & 138 & 138 & 138 & 138 \\
Mínimo & 1 & 0 & 1 & 0 & 0 & 0 & 0 & 0 & 0 & 0 & 0 & 0 \\
Máximo & 42 & 10 & 29 & 162 & 90 & 90 & 70 & 80 & 100 & 100 & 80 & 100 \\
Media & 14,0 & 3,3 & 25,0 & 3,0 & 5,3 & 4,3 & 7,4 & 3,9 & 36,7 & 34,7 & 6,3 & 14,5 \\
Varianza (n-1) & 88,8 & 4,6 & 7,3 & 256 & 312 & 126 & 217 & 100 & 1688 & 2154 & 211 & 733 \\
Desviación típica (n-1) & 9,4 & 2,1 & 2,7 & 16,0 & 18 & 11 & 14,7 & 10 & 41 & 46,4 & 14,5 & 27,1 \\
\hline
\end{tabular}

Nota: Prof $(\mathrm{m})$ - profundidad en metros; Vis (m)- visibilidad en metros; Tem $\left({ }^{\circ} \mathrm{C}\right)$ - temperatura en grados Celsius; Num org- número de organ ismos censados; Pas- pastos; Alg- algas; Esp- esponjas; Are- arena; Ped- Pedacería (restos de rocas y corales); CM- coral muerto 
Tabla 3. Prueba de normalidad de Anderson-Darling aplicada a los datos obtenidos durante el muestreo de pepino de mar / AndersonDarling normality test applied to data obtained during sampling of sea cucumber

\begin{tabular}{lcc}
\hline \multicolumn{3}{c}{ Prueba de normalidad de Anderson-Darling $(P=0,05)$} \\
\hline \multicolumn{1}{c}{ Variable } & $\begin{array}{c}\text { Estadístico } \\
\left(\mathrm{A}^{2}\right)\end{array}$ & Probabilidad \\
\hline Profundidad & 1,56 & 0,004 \\
Visibilidad & 6,55 & $<0,001$ \\
Temperatura & $>$ infinito & $<0,001$ \\
Número de organismos & 41,39 & $<0,001$ \\
Pastos & 41,27 & $<0,001$ \\
\hline Algas & 29,14 & $<0,001$ \\
Coral & 25,76 & $<0,001$ \\
Esponjas & 28,87 & $<0,001$ \\
Arena & 12,90 & $<0,001$ \\
Limo & 24,40 & $<0,001$ \\
Pedacería & 28,87 & $<0,001$ \\
Coral muerto & 25,17 & $<0,001$ \\
\hline
\end{tabular}

\section{Discusión}

Los resultados obtenidos muestran una baja densidad poblacional de las dos especies de pepino de mar analizadas en este trabajo para el SAV durante las tres temporadas de muestreo. En el caso de I. badionotus de la Península de Yucatán, el INAPESCA ha establecido como puntos de referencia una biomasa de 3000 ton, una densidad promedio de 0,025 ind. $\mathrm{m}^{-2}$ y una talla mínima de $23 \mathrm{~cm}$ de longitud, lo cual permite mantener en buen estado la población (INAPESCA 2012 $2^{1}$, por lo que la densidad promedio obtenida en este estudio está por debajo de los límites señalados. Bell et al. (2008) sugieren que la densidad promedio en las poblaciones de pepino de mar debe ser de 0,001 a 0,005 ind. $\mathrm{m}^{-2}$ para evitar efectos de depensación, agrupados de 10 o más organismos y separados de 5 a $10 \mathrm{~m}$ de distancia entre sí. Los resultados de esta investigación indicaron que la población de las especies identificadas de pepino de mar en el SAV, no cubre estos parámetros, lo cual determina que este recurso no se encuentra en cantidad suficiente para sustentar una pesquería o bien, que estos organismos se encuentran sometidos a la pesca furtiva, que derivaría en una sobrepesca y es necesario disminuir esta actividad a través de una mayor vigilancia y monitoreo por parte de las autoridades dentro del área marina protegida.

Tabla 4. Coeficientes de correlación ( $r$ ) entre las variables registradas durante los muestreos y la presencia de pepino de mar / Correlation coefficients $(r)$ between the variables recorded during sampling and the presence of sea cucumber

\begin{tabular}{lccccccccccc}
\hline & $\begin{array}{c}\text { Núm } \\
\text { org. }\end{array}$ & $\begin{array}{c}\text { Vis } \\
(\mathrm{m})\end{array}$ & $\begin{array}{c}\text { Tem } \\
\left({ }^{\circ} \mathrm{C}\right)\end{array}$ & Pas & Alg & Coral & Esp & Are & Limo & Ped & $\begin{array}{c}\text { CM } \\
\text { Prof } \\
(\mathrm{m})\end{array}$ \\
\hline Núm org. & & & & & & & & & & & \\
Visibilidad $(\mathrm{m})$ & $-0,1$ & & & & & & & & & & \\
Temperatura $\left({ }^{\circ} \mathrm{C}\right)$ & 0,2 & $-0,1$ & & & & & & & & & \\
Pastos & 0,6 & 0,0 & 0,2 & & & & & & & & \\
Algas & 0,3 & $-0,1$ & 0,1 & 0,4 & & & & & & & \\
Coral & 0,1 & 0,1 & 0,1 & $-0,1$ & 0,4 & & & & & & \\
Esponjas & 0,2 & 0,1 & 0,1 & $-0,1$ & 0,2 & 0,7 & & & & & \\
Arena & 0,3 & $-0,1$ & 0,3 & 0,4 & 0,2 & 0,0 & 0,1 & & & & \\
Limo & $-0,3$ & $-0,1$ & $-0,2$ & $-0,3$ & $-0,3$ & $-0,3$ & $-0,3$ & $-0,7$ & & & \\
Pedacería & 0,4 & 0,0 & 0,2 & 0,4 & 0,6 & 0,4 & 0,4 & 0,2 & $-0,4$ & & \\
Coral muerto & 0,1 & 0,1 & 0,1 & $-0,1$ & 0,3 & 0,8 & 0,7 & 0,1 & $-0,4$ & 0,3 & \\
Profundidad $(\mathrm{m})$ & $-0,5$ & $-0,1$ & $-0,3$ & $-0,4$ & $-0,3$ & $-0,2$ & $-0,2$ & $-0,2$ & 0,5 & $-0,4$ & $-0,2$ \\
\hline
\end{tabular}

Nota: La pedacería corresponde a restos de rocas y corales 
En la comparación de las densidades obtenidas por banco (Tabla 6), se muestra que existe heterogeneidad tanto en el número de transectos como en el promedio de densidad en los diferentes bancos donde se realizó el muestreo. A pesar de que el área muestreada en el banco $1\left(20.714 \mathrm{~m}^{2}\right)$, donde se realizaron 44 transectos, es mayor en relación al banco 10 $\left(4.180 \mathrm{~m}^{2}\right)$ en la cual sólo se realizaron 13 transectos, se obtuvo una menor cantidad de estaciones donde se encontró pepino de mar (solo 5 ejemplares), con respecto al último, en el que se localizaron 12 estaciones con presencia de este organismo, con un promedio de 0,071 $\pm 0,080$ ind. $\mathrm{m}^{-2}$. No obstante, de acuerdo con López-Rocha (2011) y Espinoza-Méndez (com. pers. J Espinoza ${ }^{8}$ ), por las dimensiones del banco 10 (700 m de largo y 100 de ancho aproximadamente) no debe considerarse como un sitio con potencial pesquero, ya que el área no sería capaz de mantener los puntos de referencia límites establecidos para la pesquería de este recurso; el lugar debe considerarse un parche o sitio de agregación de pepinos de mar en el SAV. Particularmente este banco se encuentra pegado a la línea de costa en el arrecife Ingenieros del SAV (DOF 2012) $)^{4}$.

Tabla 5. Estimación de la abundancia promedio y poblacional (bancos) de pepino de mar en el Sistema Arrecifal Veracruzano / Average estimated abundance and population (banks) of sea cucumber in Sistema Arrecifal Veracruzano

\begin{tabular}{cccccc}
\hline Banco & $\begin{array}{c}\text { Área del } \\
\text { banco }\left(\mathrm{m}^{2}\right)\end{array}$ & $\begin{array}{c}\text { Área } \\
\text { muestreada }\left(\mathrm{m}^{2}\right)\end{array}$ & $\begin{array}{c}\text { Número de } \\
\text { individuos }\end{array}$ & $\begin{array}{c}\text { Densidad } \\
\text { promedio } \\
\left(\text { ind. } \mathrm{m}^{-2}\right)\end{array}$ & $\begin{array}{c}\text { Densidad } \\
\text { poblacional } \\
\left(\text { ind. } \mathrm{m}^{-2}\right)\end{array}$ \\
\hline Banco 01 & 34.354 .288 & 20.714 & 8 & $3,86 \times 10^{-4}$ & $2,33 \times 10^{-7}$ \\
Banco 02 & 11.189 .906 & 1.400 & 0 & 0 & 0 \\
Banco 03 & 6.307 .201 & 3.000 & 2 & $6,67 \times 10^{-4}$ & $3,17 \times 10^{-7}$ \\
Banco 05 & 1.473 .904 & 2.000 & 0 & 0 & 0 \\
Banco 06 & 1.474 .198 & 3.600 & 11 & 0,003 & $7,46 \times 10^{-6}$ \\
Banco 08 & 1.649 .361 & 2.900 & 5 & 0,002 & $3,03 \times 10^{-6}$ \\
Banco 10 & 58.065 & 4.180 & 282 & 0,067 & 0,005 \\
Total & $77.756 .584,02$ & 37.794 & 308 & 0,008 & $3,96 \times 10^{-6}$ \\
\hline
\end{tabular}

Considerando el enfoque precautorio (FAO 1996), la actividad pesquera en esta área hace vulnerable al recurso, ya que es de muy fácil acceso (caminando desde la playa y la recolección del pepino se realizaría de la misma manera ya que la profundidad promedio se encuentra entre 0,3 y $2 \mathrm{~m}$ ), lo que podría llevar a una sobreexplotación en este sitio. Con el objetivo de obtener un permiso de pesca de fomento de pepino de mar, en el 2012, un grupo de pescadores realizaron muestreos en los puntos provenientes de las bitácoras de pesca dentro del SAV (principalmente en el banco 02), donde se reportaban capturas de 600 y 700 organismos a profundidades menores a $3 \mathrm{~m}$. Los resultados de esta investigación permitieron constatar que la profundidad no correspondía con lo reportado (estaciones de 2 a $3 \mathrm{~m}$ de profundidad mencionadas en bitácora, presentaron profundidades entre 20 y 30 m) y no se registró una gran abundancia del recurso ya que de acuerdo a lo establecido por CONAPESCA (2013) ${ }^{9}$, el volumen de producción pesquera fue de 2.649 ton de peso desembarcado reportado únicamente para el estado de Yucatán, lo cual representa el 95,9\% de volumen nacional total, por lo que la evaluación realizada en el 2012, no explica la cantidad de captura.

Para el caso particular del SAV y la complejidad de sus arrecifes coralinos, se han establecido puntos de referencia para el aprovechamiento de $H$. floridana considerando el principio de enfoque precautorio (FAO 1996). La cuota de captura máxima establecida fue de 34,67 ton, una densidad final de organismos post-pesca de 0,12 ind. $\mathrm{m}^{-2}$ y una talla mínima de $22 \mathrm{~cm}$, para que la población estimada pueda sostener la actividad pesquera. De acuerdo con los resultados del presente estudio, ninguno de los bancos evaluados cumple estos parámetros, por lo que no se recomienda el aprovechamiento pesquero en los sitios propuestos.

Tabla 6. Estimación de los parámetros obtenidos para la comparación de densidades entre bancos de pepino de mar en el Sistema Arrecifal Veracruzano / Estimated parameters for density comparation of sea cucumber's banks in Sistema Arrecifal Veracruzano

\begin{tabular}{|c|c|c|c|c|c|c|c|}
\hline Banco & $\begin{array}{c}\text { Densidad promedio } \\
\text { (ind. } \mathrm{m}^{-2} \text { ) }\end{array}$ & $\bar{x}$ & se & Ic L & Ic $U$ & $\mathrm{n}$ & $\mathrm{m}$ \\
\hline Banco 01 & $3,86 \times 10^{-4}$ & $4,83 \times 10^{-4}$ & $2,20 \times 10^{-4}$ & $1,14 \times 10^{-4}$ & $8,52 \times 10^{-4}$ & 44 & 5 \\
\hline Banco 02 & 0 & 0 & 0 & 0 & 0 & 7 & 0 \\
\hline Banco 03 & $6,67 \times 10^{-4}$ & $7,14 \times 10^{-4}$ & $4,85 \times 10^{-4}$ & $1,40 \times 10^{-4}$ & $1,57 \times 10^{-3}$ & 14 & 2 \\
\hline Banco 05 & 0 & 0 & 0 & 0 & 0 & 10 & 0 \\
\hline Banco 06 & 0,003 & $2,34 \times 10^{-3}$ & $1,40 \times 10^{-3}$ & $9,5 \times 10^{-5}$ & $4,79 \times 10^{-3}$ & 16 & 3 \\
\hline Banco 08 & 0,002 & $3,33 \times 10^{-3}$ & $3,33 \times 10^{-3}$ & 0,002 & 0,009 & 15 & 1 \\
\hline Banco 10 & 0,067 & 0,070 & 0,022 & 0,031 & 0,110 & 13 & 12 \\
\hline Total & 0,008 & 0,008 & 0,003 & 0,003 & 0,014 & 119 & 23 \\
\hline
\end{tabular}

${ }^{8}$ Espinoza-Méndez J. Investigador del Centro Regional de Investigación Pesquera, Yucalpetén, Yucatán. Instituto Nacional de Pesca. ${ }^{9}$ CONAPESCA. 2013. Anuario estadístico de acuacultura y pesca 2012, 385 pp. <http://www.conapesca.gob.mx/work/sites/cona/ dgppe/2012/ANUARIO_ESTADISTICO_DE_ACUACULTURA_Y_PESCA_2012.pdf> 


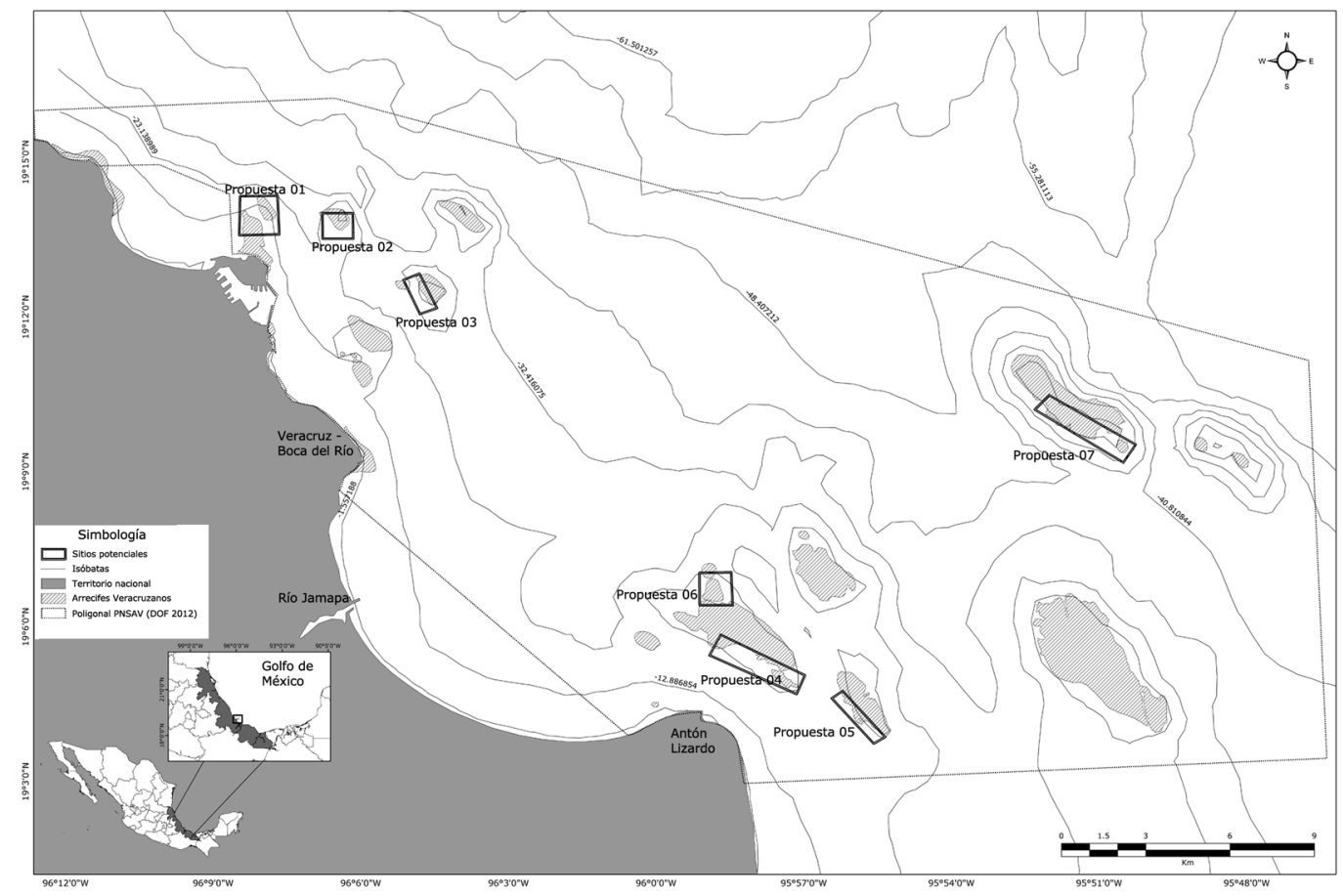

Figura 3. Propuesta de sitios potenciales con existencia de pepino de mar en el SAV, considerando los límites en cuanto a distribución y los sitios donde se observaron especímenes / Proposed potential sites with presence of sea cucumber in SAV, considering the limits in terms of distribution and sites where specimens were observed

La evaluación geoestadística, no fue posible realizarla debido a que las bajas abundancias (menos de 100 observaciones para un Kriging aceptable), no permitieron interpolar la distribución de las especies, ya que el recurso no se distribuye de manera homogénea en los bancos evaluados, lo cual no fue posible encontrar una autocorrelación espacial (Webster \& Oliver 2007, Anderson et al. 2011). Los resultados obtenidos muestran que la población de pepino en el SAV se encuentra fragmentada y con baja densidad, locual indica que no es una población virgen y que es necesario establecer medidas, regulaciones precautorias, aplicación de la ley y un mayor compromiso de los pescadores y autoridades (Purcell 2010), que permitan la recuperación del recurso en la zona. No obstante, al ser la primera evaluación prospectiva de pepino de mar realizada en el SAV, no fue posible determinar si la distribución del recurso es debido al efecto de la pesca (legal y/o furtiva) (JiménezBadillo 2008) u otros factores ambientales y ecológicos que inciden en este sistema arrecifal (Ortíz-Lozano 2012). De todos modos, es necesario establecer medidas regulatorias para recuperar la población de pepino de mar en el SAV para posteriormente evaluar el potencial pesquero de este recurso. Recientemente, se están realizando esfuerzos para los cultivos de I. badionotus y $H$. floridana en el Golfo de México (Rodríguez-Serna et al. 2012), lo que representa una oportunidad en un futuro para repoblar y aprovechar de manera sustentable este recurso en Veracruz.

Con base en lo anterior, se proponen algunos bancos como 'sitios potenciales' (Purcell 2010), con presencia de pepino de mar dentro del SAV (Fig. 3). Estas áreas se restringen a la laguna y pendiente arrecifal de la zona de sotavento (Lara et al. 1992) de algunos arrecifes, delimitado a la isobata de $20 \mathrm{~m}$, debido a que más allá de esa profundidad no se encontró ningún organismo en esta investigación. Estos sitios potenciales deberán ser monitoreados para observar si la población se recupera y en un futuro sea susceptible a explotación, no obstante esta primera aproximación contribuye al conocimiento de los patrones de abundancia y densidad de la situación del recurso pepino de mar en Veracruz, hasta ahora muy poco estudiadas en el SAV.

\section{Agradecimientos}

Se agradece a la Comisión Nacional de Áreas Marinas Protegidas (CONANP) por su colaboración durante algunos muestreos de campo, así como a los buzos, técnicos e investigadores del INAPESCA y de la Universidad Veracruzana que apoyaron durante los muestreos realizados. Al M. E. P. Heber Zea por su apoyo para el análisis estadístico. 


\section{LITERATURA CITADA}

Anderson D, D Sweeney \& T Williams. 2008. Estadística para administración y economía, 1060 pp. Editorial Cengage Learning, México D.F.

Anderson S, J Mills-Flemming, R Watson \& H Lotze. 2011. Serial exploitation of global sea cucumber sheries. Fish and Fisheries 12:317-339.

Bell JM, K Leber, H Lee-Blankenship, N Loneragan \& R Masuda. 2008. A new era for restocking, stock enhancement and sea ranching of coastal fisheries resources. Reviews in Fisheries Science 16(1-3): 1-9.

Cadima, EL, A Caramelo, M Afonso-Dias, P Conte de Barros, M Tandstad \& J de Leiva-Moreno. 2005. Sampling methods applied to fisheries science: a manual. FAO Fisheries Technical Paper 434: 1-88.

Cervera-Cervera K, J Espinoza-Méndez, D De AndaFuentes \& R Burgos-Rosas. 2008. Avances en el conocimiento de la distribución, abundancia y disponibilidad pesquera del recurso pepino de mar en el Estado de Yucatán. En: Espino E, MA Carrasco, P Fuentes, EG Cabral, M Puente \& A García (eds). IV Foro Científico de Pesca Ribereña, Memorias, pp: 11-12. Instituto Nacional de Pesca, Acapulco.

Cochran W. 1977. Sampling techinques, 442 pp. John Wiley \& Sons Press, New York.

Conand C. 2006. Sea cucumber biology: taxonomy; distribution; biology; conservation status. In: Bruckner AW (ed). Proceedings of the CITES workshop on the conservation of sea cucumbers in the families Holothuriidae and Stichopodidae. NOAATechnical Memorandum, NMFS-OPR 34:33-50.

Desola-Alà J. 1990. Accidentes de buceo (1). Enfermedad descompresiva. Medicina Clínica 95(4): 147-156.

Durán-González A, A Laguarda-Figueras, FA Solís-Marín, BE Buitrón Sánchez, C Gust Ahearn \& J Torres-Vega. 2005. Equinodermos (Echinodermata) de las aguas mexicanas del Golfo de México. Revista de Biología Tropical 53(3): 53-68.

Fajardo-León M, M Suárez-Higuera, A del ValleManríquez \& A Hernández-López. 2008. Biología reproductiva del pepino de mar Parastichopus parvimensis (Echinodermata: Holothuroidea) de Isla Natividad y Bahía Tortugas, Baja California Sur, México. Ciencias Marinas 34(2): 165-177.

FAO. 1996. Precautionary approach to capture fisheries and species introductions. FAO Technical Guidelines for Responsible Fisheries 2: 1-54.

Flowes-Cano RS, RJ Flowers \& F Rivera-Trejo. 2014. Evaluación de criterios de selección de modelos probabilísticos: validación con series de valores máximos simulados. Tecnología y Ciencias del Agua 5(5): 189-197.

Gutiérrez de Velasco G \& CD Winant. 1996. Seasonal patterns of wind stress and wind stress curl over the Gulf of Mexico. Journal of Geophysical Research 101(C8): 127-140.
Herrero-Pérezrul MD \& H Reyes-Bonilla. 2008. Weight length relationship and relative condition of the holothurians Isostichopus fuscus at Espíritu Santo Island, Gulf of California, México. Revista de Biología Tropical 56(3): 273280.

Herrero-Pérezrul MD, H Reyes-Bonilla, F GarcíaDomínguez \& CE Cintra-Buenrostro. 1999. Reproduction and growth of Isostichopus fuscus (Echinodermata: Holothuroidea) in the southern Gulf of California, México. Marine Biology 135: 521-532.

Hill J \& C Wilkinson. 2004. Methods for ecological monitoring of coral reefs: A resource for managers, 123 pp. Australian Institute of Marine Science, Townsville.

Jiménez-Badillo ML. 2008. Management challenges of smallscale shing communities in a protected reef system of Veracruz, Gulf of Mexico. Fisheries Management and Ecology 15: 19-26.

Krutak PR. 1997. Petrography and provenance of siliclastic sediments, Veracruz-Antón Lizardo Reefs, México. In: SanJoon J \& Y Hi-Il. (eds). Paleoceanography and paleoclimatology in the northwest Pacific region. Special Issue 3, Ocean Research 19: 231-243.

Lara M, C Padilla, C García \& J Espejel. 1992. Coral Reef of Veracruz Mexico I. Zonation and Community. In: Richmond RH (ed). Proceedings of the $7^{\text {th }}$ International Coral Reef Symposium, University of Guam Marine Laboratory, Guam, pp. 535-544.

López-Rocha J. 2011. Distribución y abundancia del pepino de mar Isostichopus badionotus frente a la costa de Sisal Yucatán. Proceedings of the Gulf and Caribbean Fisheries Institute 64: 153-160.

Martella M, E Trumper, L Bellis, D Renison, P Giordano, G Bazzano \& R Gleiser. 2012. Manual de ecología poblaciones: Introducción a las técnicas para el estudio de las poblaciones silvestres. Reduca (Biología), Serie Ecología 5(1): 1-31.

Martínez-Ortega RM, L Tuya-Pendás, M Martínez-Ortega, A Pérez-Abreu \& AM Cánovas. 2009. El coeficiente de correlación de los rangos de Spearman Caracterización. Revista Habanera de Ciencias Médicas de la Habana 3(2): $1-20$.

Moral-García FJ. 2004. Aplicación de la geoestadística en las ciencias ambientales. Ecosistemas 13(1): 78-86.

Navarro P, S García-Sanz \& F Tuya. 2013. Patrones de abundancia y talla de Holothuria sanctori, Holothuria mammata y Holothuria arguinensis (Echinodermata: Holoturoidea) en la isla de Gran Canaria, Atlántico oriental. Revista de Biología Marina y Oceanografía 48(2): 273-284.

Ortíz-Lozano L. 2012. Identification of priority conservation actions in marine protected areas: Using a causal networks approach. Ocean \& Coastal Management 55: 74-83.

Purcell SW. 2010. Manejo de las pesquerías de pepino de mar con un enfoque ecosistémico. FAO Documento Técnico de Pesca y Acuacultura 520: 1-169. 
Purcell SW, H Gossuin \& N Agudo. 2009. Status and management of the sea cucumber fishery of La Grande Terre, New Caledonia, 136 pp. World Fish Center Studies and Reviews No. 1901. The World Fish Center, Penang.

Purcell SW, Y Samyn \& C Conand. 2012. Commercially important sea cucumbers of the world. FAO Species Catalogue for Fishery Purposes 6: 1-150.

Ramírez-González A. 2006. Ecología: Métodos de muestreo y análisis de poblaciones y comunidades, 265 pp. Pontificia Universidad Javeriana, Bogotá.

Ramofafia C, S Battaglene, J Bell \& M Byme. 2000. Reproductive biology of commercial sea cumber Holothuria fuscogilva in the Salomon Islands. Marine Biology 136: 10451056.

Reyes-Bonilla H \& MD Herrero-Pérezrul. 2003. Population parameters of an exploited population of Isostichopus fuscus (Holothuroidea) in the southern Gulf of California, Mexico. Fisheries Research 59: 423-430.

Rodríguez-Gil L. 2007. El pepino de mar como un recurso potencial de la costa del Estado de Yucatán, México. Proceedings of the Gulf and Caribbean Fisheries Institute 59:289-296.

Rodríguez-Serna M, C Carmona-Osalde, X Guzmán-García \& E Puerto-Novelo. 2012. Primeras experiencias para el desarrollo del cultivo de holoturidos (Isostichopus badionotus y Holothuria floridana) endémicos del Golfo de México. AquaTIC 36: 3-10.

Salas-Pérez J \& A Granados-Barba. 2008. Oceanographic characterization of the Veracruz reefs system. Atmósfera 21(3): 281-301.

Salas-Pérez J \& V Arenas-Fuentes. 2011. Winter water mass of the Veracruz Reef System. Atmosfera 24(2): 221-231.

Singh-Cabanillas J \& A Vélez-Barajas. 1996. La pesquería del pepino de mar Isostichopus fuscus en la costa oriental de Baja California Sur y propuestas de regulación. Ciencia Pesquera 12: 13-18.

Skewes TD, D Dennis, T Wassenberg, M Austin, C Moeseneder \& A Koutsoukos. 2002. Surveying the distribution and abundance of Holothuria scabra (sandfish) in Moreton Bay. CSIRO Division of Marine Research Final Report, 20 pp.
Solís-Marín FA, A Laguarda Figueras \& MA Gordillo Hernández. 2007. Estudio taxonómico de los equinodermos del Parque Nacional Sistema Arrecifal Veracruzano. En: Granados-Barba A, LG Abarca-Arenas \& JM VargasHernández (eds). Investigaciones Científicas en el Sistema Arrecifal Veracruzano, pp. 73-100. Universidad Autónoma de Campeche, Campeche.

Solís-Marín FA, M Honey-Escandón, M Herrero-Perezrul, F Benitez-Villalobos, J Díaz-Martínez, B BuitrónSánchez, J Palleiro-Nayar \& A Durán-González. 2013. The Echinoderms of Mexico: Biodiversity, distribution and current state of knowledge. En: Alvarado JJ \& FA SolísMarín (eds). Echinoderm research and diversity in Latin America, pp: 11-65. Springer-Verlag, Berlin / Heidelberg.

Solís-Marín FA, A Laguarda-Figueras \& M HoneyEscandón. 2014. Biodiversidad de equinodermos (Echinodermata) en México. Revista Mexicana de Biodiversidad 85: 441-449.

Stephens MA. 1974. EDF statistics for goodness of fit and some comparisons. Journal of the American Statistical Association 69(347): 730-737.

Tunnell J Jr, E Chávez \& K Withers. 2007. Coral reefs of the southern Gulf of Mexico, 360 pp. Texas A\&M University Press, Texas.

Tuz-Sulub A \& A Aguilar-Perera. 2011. Aprovechamiento del pepino de mar: pesquería potencial para el desarrollo económico y social en la costa norte de la Península de Yucatán. Bioagrociencias 4(2): 17-22.

Uthicke S. 2001. Nutrient regeneration by abundant coral reef holothurians. Journal of Experimental Marine Biology and Ecology 265: 153-170.

Webster R \& MA Oliver. 2007. Geostatistics for environmental scientists, 332 pp. John Wiley \& Sons, Chichester.

Zavala-Hidalgo J, S Morey \& J O'Brien. 2003. Seasonal circulation on the western shelf of the Gulf of Mexico using a high-resolution numerical model. Journal of Geophysical Research (108)12: 1-19.

Recibido el 7 de octubre de 2016 y aceptado el 8 de agosto de 2017

Editor: Claudia Bustos D. 